\title{
Uso de substâncias na adolescência e problemas familiares
}

\author{
Adolescent substance use and \\ family problems
}

\author{
1 Faculdade de Medicina, \\ Universidade de São Paulo, \\ São Paulo, Brasil. \\ Correspondência \\ A. Malbergier \\ Departamento de Psiquiatria \\ Faculdade de Medicina, \\ Universidade de São Paulo. \\ Rua Dr. Ovídio Pires de \\ Campos 785, São Paulo, SP \\ 05403-010, Brasil. \\ amalbergier@uol.com.br
}

\begin{abstract}
This study aimed to evaluate the association between substance use (alcohol, tobacco, and illicit drugs) and family problems among 965 adolescents from 50 public schools in two cities in São Paulo State, Brazil, in 2007. The Drug Use Screening Inventory (DUSI) was used for data collection. Use of alcohol, tobacco, and illicit drugs was associated with a negative assessment of the family relationship, lack of monitoring/support, and psychoactive substance use by family members $(p<0.05)$. Adolescents that reported having used alcohol, tobacco, and illicit drugs had more family problems than those who did not consume any substance $(p<0.001)$. Adolescents that used alcohol and tobacco $(p=0.028)$ and illicit drugs $(p<0.001)$ reported having more family problems than those who used only alcohol. The results highlight the importance of awareness of alcohol and tobacco use by adolescents, since such use was associated with significant family impairments, similar to illicit drug use.
\end{abstract}

Alcoholic Beverages; Tobacco; Street Drugs; Family Relations; Adolescent
André Malbergier 1

Luciana Roberta Donola Cardoso 1

Ricardo Abrantes do Amaral 1

\section{Introdução}

O consumo de substâncias psicoativas é um grave problema de saúde pública. O início do uso geralmente ocorre na adolescência e, nesta fase, tem sido associado a problemas escolares (faltas, repetência, evasão escolar e dificuldade de aprendizagem), sociais (relacionamentos com outros usuários e envolvimento em atividades ilegais), características de personalidade (intolerância à frustração, desinibição, agressividade e impulsividade), transtornos psiquiátricos e problemas familiares 1,2,3.

Os estudos têm mostrado que variáveis relacionadas ao ambiente familiar exercem grande influência no início e na manutenção do consumo de álcool, tabaco e maconha entre adolescentes 4,5 . Fatores como relacionamento ruim com os pais, ter membro da família que abusa e/ ou é dependente de alguma substância, violência doméstica, desorganização familiar, viver apenas com um dos pais, pouca comunicação entre familiares e falta de suporte e monitoramento familiar têm sido associados ao uso de álcool, tabaco e outras drogas nessa fase da vida 6,7,8,9. Embora essas associações sejam conhecidas na literatura especializada, os estudos, todavia, não avaliam se o consumo de diferentes drogas estaria associado a vários níveis de problemas familiares.

Este tema também merece atenção em nosso país já que, no momento atual, o Brasil parece 
apresentar uma tendência de aumento de consumo de drogas (lícitas e ilícitas). Os trabalhos científicos nacionais publicados sobre o uso de drogas entre adolescentes e as associações com variáveis familiares analisaram somente uma droga (por exemplo, álcool) 10 ou os problemas familiares foram apresentados como mais uma variável estudada sem se aprofundar no tema 11 .

Os objetivos deste estudo são: (a) avaliar a associação entre variáveis do sistema familiar e o consumo de álcool, tabaco e drogas ilícitas; (b) avaliar a possível associação de diferentes padrões de consumo de drogas (uso de álcool, uso de tabaco, uso de álcool e tabaco e uso de drogas ilícitas) com diferentes níveis de problemas familiares.

\section{Método}

\section{Desenho e população do estudo}

Este trabalho, de desenho seccional, foi realizado em 50 escolas públicas estaduais dos municípios de Jacareí e Diadema (São Paulo, Brasil) que ofereciam o Programa Escola da Família (PEF). Esse programa é uma iniciativa da Secretaria da Educação do Estado de São Paulo com a cooperação da UNESCO (Organização das Nações Unidas para a Educação, Ciência e Cultura) e consiste em utilizar o espaço físico das escolas públicas estaduais, nos finais de semana, para desenvolver atividades socioeducativas com os jovens e suas famílias.

A seleção das escolas foi realizada junto com os coordenadores do PEF e com os coordenadores da Secretaria da Educação do Estado de São Paulo. Buscou-se, nessa seleção, a indicação de escolas que estivessem na média, entre as escolas do estado, em relação a desempenho, uso de drogas e violência. Com isso, 50 escolas foram selecionadas. Após a avaliação do consumo de drogas, os adolescentes participavam de um programa de prevenção de drogas. Para esse programa, o número de adolescentes deveria ser de 20. Assim, chegou-se a amostra de 1.000 sujeitos nesta pesquisa.

Todos os alunos que faziam parte do PEF foram convidados a participar da pesquisa até que se atingisse o número necessário para aquela escola. Os critérios de inclusão no trabalho eram: ter idade entre 10 e 18 anos, participar desse programa nas escolas selecionadas e trazer o termo de consentimento assinado pelos pais ou responsáveis.

\section{Procedimentos}

O objetivo inicial desta pesquisa era abranger 1.000 adolescentes, 20 em cada escola. Entretanto, de acordo com os critérios de inclusão - 6 estudantes foram excluídos por terem menos de 10 ou mais de 18 anos - e do prazo estipulado para a coleta de dados, conseguiu-se avaliar 965 adolescentes. A pesquisa foi realizada em 2007.

Inicialmente, o projeto foi divulgado por meio de cartazes afixados na escola. Esses cartazes continham informações sobre a realização da pesquisa e o que era necessário fazer para participar. Os interessados retiravam o termo de consentimento, levavam-no aos pais e/ou responsáveis e o devolvia ao monitor antes da aplicação do questionário. Todos os adolescentes e os seus responsáveis assinaram o termo de consentimento, garantindo a participação voluntária, sigilosa e o cumprimento das normas éticas de pesquisa em seres humanos.

Para os estudantes que trouxeram o termo de consentimento, os monitores agendaram dia e horário para a aplicação do questionário. Eles recebiam o questionário da pesquisa que é autoaplicável, respondiam às perguntas na presença do monitor e o inseriam numa urna sem precisar se identificar.

Para aplicação desse instrumento, os autores treinaram 50 monitores do PEF.

Este projeto foi aprovado pela Comissão de Ética do Hospital das Clínicas da Faculdade de Medicina da Universidade de São Paulo (processo $425 / 06)$.

\section{Instrumento e variáveis de estudo}

Nesta pesquisa, utilizou-se o questionário autoaplicável DUSI (Drug Use Screening Inventory) já traduzido e validado no Brasil 12,13. Esse instrumento contém questões que buscam investigar o consumo de substâncias no mês anterior à entrevista e fatores relacionados ao uso. Os adolescentes responderam a todo o questionário, mas para este artigo foram analisadas as áreas do consumo (área I) e de problemas familiares (área VI e parte da $\mathrm{X}$ ), além dos dados sociodemográficos contidos no questionário.

\section{- Uso de substâncias}

O questionário avalia a frequência do consumo de álcool, tabaco e drogas ilícitas (anfetamina, ecstasy, cocaína, crack, maconha, alucinógenos, tranquilizantes, ansiolíticos, esteróides, inalantes e solventes) nos trinta dias anteriores à entrevista. Foram consideradas ilícitas algumas drogas lícitas (por exemplo, anfetaminas) usa- 
das de forma ilícita (sem prescrição ou indicação médica).

\section{- Variáveis do sistema familiar}

As questões abordavam a avaliação do relacionamento com os pais, atenção, suporte, monitoramento e uso de álcool e outras drogas por membros da família. Seguem as questões relacionadas a problemas familiares do DUSI:

1. Algum membro de sua família (mãe, pai, irmão ou irmã) usou maconha ou cocaína no último ano?

2. Algum membro de sua família usou álcool a ponto de causar problemas em casa, no trabalho ou com amigos?

3. Algum membro de sua família foi preso no último ano?

4. Você tem discussões frequentes com seus pais ou responsáveis que envolvam gritos e berros?

5. Sua família dificilmente faz coisas juntas?

6. Seus pais ou responsáveis desconhecem o que você gosta e o que não gosta?

7. Na sua casa faltam regras claras sobre o que você pode e não pode fazer?

8. Seus pais ou responsáveis desconhecem o que você realmente pensa ou sente sobre as coisas que são importantes para você?

9. Seus pais ou responsáveis brigam muito entre si?

10. Seus pais ou responsáveis frequentemente desconhecem onde você está ou o que você está fazendo?

11. Seus pais ou responsáveis estão fora de casa a maior parte do tempo?

12. Você sente que seus pais ou responsáveis não se importam ou não cuidam de você?

13. Você se sente infeliz em relação ao local no qual você vive?

14. Você se sente em perigo em casa?

15. Na maioria das festas que você tem ido recentemente, os pais estão ausentes?

16. Nas suas horas livres você simplesmente passa a maior parte do tempo com amigos?

17. Você realiza a maior parte das atividades de lazer sozinho?

As questões eram respondidas com "SIM" ou "NÃO".

\section{Análise dos dados}

Nas análises univariadas, empregou-se o teste qui-quadrado com correção de Yates quando necessário (valor esperado menor que 5) para as variáveis categóricas e o teste de Kruskal-Wallis com correção de Bonferroni para as variáveis contínuas. Para as análises múltiplas, foi aplicado o teste de regressão logística multinomial. O método de seleção utilizado foi o Wald's backward test.

O consumo de substância foi avaliado considerando cinco categorias: (1) não usou nenhuma substância; (2) usou apenas álcool; (3) usou apenas tabaco; (4) usou álcool e tabaco; e (5) usou drogas ilícitas, podendo ou não ter feito uso de álcool e/ou tabaco.

As análises univariadas avaliaram a relação entre as categorias de consumo de drogas e as variáveis sociodemográficas e do sistema familiar. Também foram utilizadas análises univariadas para o estudo das associações entre a média de problemas familiares e as categorias de consumo.

A análise múltipla considerou como variáveis dependentes o consumo de álcool, tabaco, álcool e tabaco, e drogas ilícitas separadamente, e como independentes as questões (listadas anteriormente) relacionadas aos problemas familiares. Também foram incluídas no modelo as covariáveis idade, gênero, pessoa com quem vive, escolaridade e repetência escolar (questões também presentes no DUSI).

Os valores descritos nos resultados e nas tabelas referem-se aos casos válidos. As respostas em branco foram excluídas das análises de cada questão.

A razão de chances (OR) foi calculada considerando um intervalo de $95 \%$ de confiança. O nível de significância foi de $5 \%$.

Para as análises utilizou-se o programa estatístico SPSS, versão 15.0 (SPSS Inc. Chicago, Estados Unidos).

\section{Resultados}

A Tabela 1 mostra a descrição da amostra. Nessa tabela estão apresentados os dados sociodemográficos e a distribuição dos problemas familiares na amostra total, que foi constituída por 965 adolescentes, $436(45,2 \%)$ do sexo feminino e 529 (54,8\%) do masculino; 831 (86,1\%) estavam no ensino fundamental, $132(13,7 \%)$ no ensino médio e dois não responderam a esta pergunta $(0,2 \%) ; 771(80,0 \%)$ nunca repetiram o ano escolar, $185(19,1 \%)$ repetiram o ano ao menos uma vez e nove não responderam (0,9\%); 606 (62,8\%) vivem com o pai e com a mãe, $218(22,6 \%)$ apenas com a mãe, $21(2,2 \%)$ apenas com o pai, 98 $(10,1 \%)$ com outros familiares, dois $(0,2 \%)$ em instituição e 20 (2,1\%) não responderam. A idade média dos adolescentes foi de 13,5 (DP = 1,41) anos.

Não houve diferenças entre os gêneros quanto à idade $(\mathrm{p}=0,670)$, escolaridade $(\mathrm{p}=0,100)$ e pessoa com que vive $(\mathrm{p}=0,923)$. O índice de 
Descrição sociodemográfica e das variáveis do sistema familiar em uma amostra de adolescentes de 50 escolas públicas estaduais de Jacareí e Diadema, São Paulo, Brasil ( $N=965)$.

\begin{tabular}{|c|c|c|}
\hline & $\mathrm{n}$ & $\%$ \\
\hline \multicolumn{3}{|l|}{ Gênero $(n=965)$} \\
\hline Meninas & 436 & 45,2 \\
\hline Meninos & 529 & 54,8 \\
\hline \multicolumn{3}{|l|}{ Idade (anos) $(n=866)$} \\
\hline $10-14$ & 653 & 75,4 \\
\hline $15-18$ & 213 & 24,6 \\
\hline \multicolumn{3}{|l|}{ Escolaridade $(n=963)$} \\
\hline Ensino Fundamental & 831 & 86,3 \\
\hline Ensino Médio & 132 & 13,7 \\
\hline \multicolumn{3}{|l|}{ Repetência escolar ( $n=956$ ) } \\
\hline Não & 771 & 80,6 \\
\hline Sim & 185 & 19,4 \\
\hline \multicolumn{3}{|l|}{ Com quem vive $(n=945)$} \\
\hline Pai e mãe & 606 & 64,1 \\
\hline Só com a mãe & 21 & 2,2 \\
\hline Só com o pai & 218 & 23,1 \\
\hline Outros familiares & 98 & 10,4 \\
\hline Instituição & 2 & 0,2 \\
\hline \multicolumn{3}{|l|}{ Relacionamento com os pais $(n=949)$} \\
\hline Bom & 815 & 85,9 \\
\hline Ruim (inclui respostas: regular e depende) & 134 & 14,1 \\
\hline \multicolumn{3}{|c|}{ Ter membro da família que usou maconha ou cocaína no último ano ( $\mathrm{n}=948)$} \\
\hline Não & 862 & 90,9 \\
\hline Sim & 86 & 9,1 \\
\hline \multicolumn{3}{|c|}{ Ter membro da família que usou álcool a ponto de causar problemas ( $n=949)$} \\
\hline Não & 742 & 78,2 \\
\hline Sim & 207 & 21,8 \\
\hline \multicolumn{3}{|c|}{ Ter membro da família que foi preso no último ano $(n=947)$} \\
\hline Não & 842 & 88,9 \\
\hline Sim & 105 & 11,1 \\
\hline \multicolumn{3}{|l|}{ Tem discussões frequentes com os pais $(n=941)$} \\
\hline Não & 749 & 79,6 \\
\hline Sim & 192 & 20,4 \\
\hline \multicolumn{3}{|l|}{ Família dificilmente faz coisas juntas $(n=945)$} \\
\hline Não & 658 & 69,6 \\
\hline Sim & 287 & 30,4 \\
\hline \multicolumn{3}{|c|}{ Pais desconhecem o que o filho gosta/desgosta $(n=936)$} \\
\hline Não & 684 & 73,1 \\
\hline Sim & 252 & 26,9 \\
\hline \multicolumn{3}{|c|}{ Faltam regras claras, em casa, sobre o que pode ou não fazer $(n=946)$} \\
\hline Não & 775 & 81,9 \\
\hline Sim & 171 & 18,1 \\
\hline \multicolumn{3}{|c|}{ Pais desconhecem o que pensa ou sente sobre coisas importantes para ele $(n=938)$} \\
\hline Não & 679 & 72,4 \\
\hline Sim & 259 & 27,6 \\
\hline
\end{tabular}

(continua) 


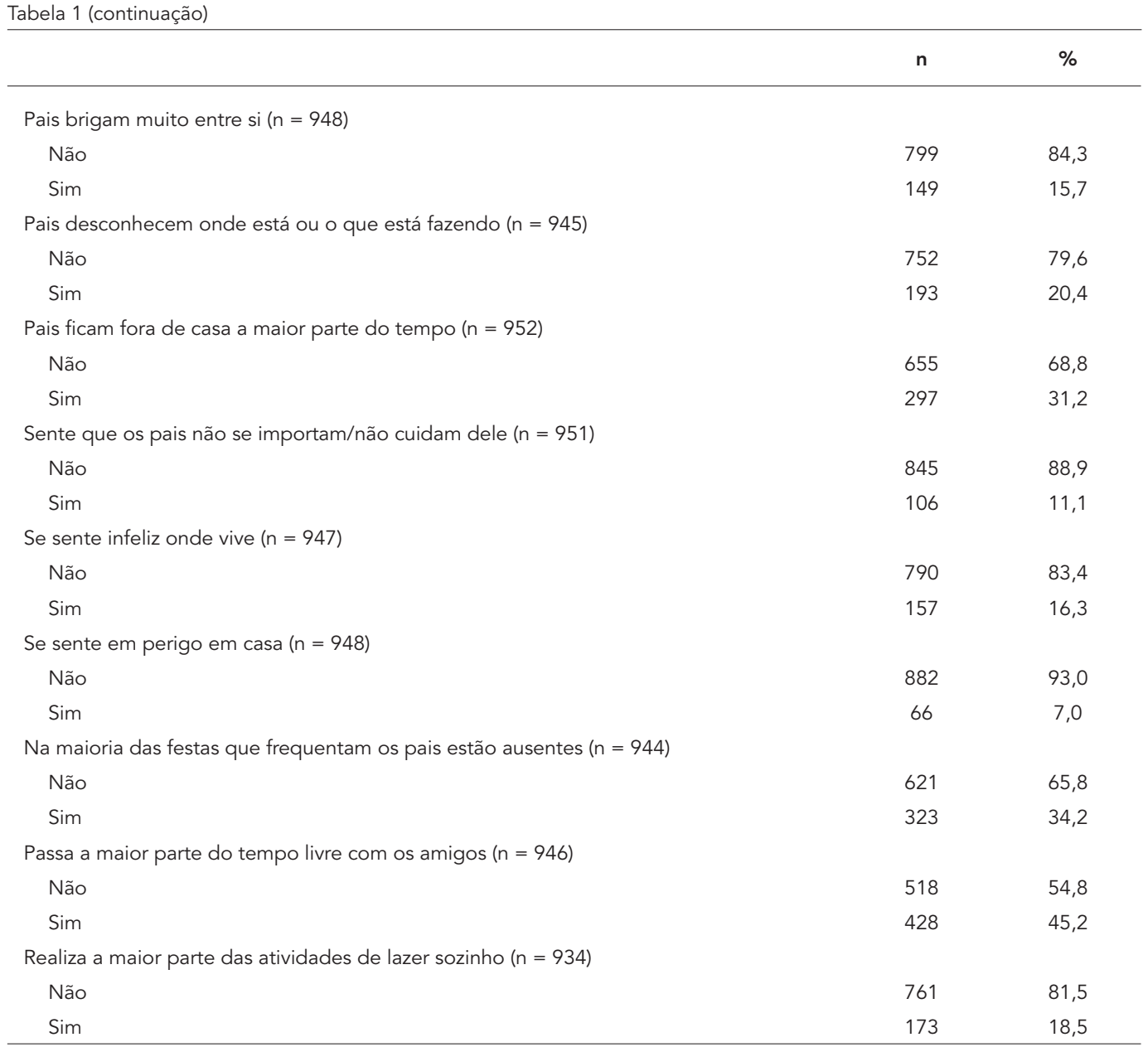

repetência escolar foi maior entre os meninos $(\mathrm{n}=126 ; 24,1 \%)$ do que entre as meninas $(\mathrm{n}=59$; 13,6\%) $(\mathrm{p}<0,001 ; \mathrm{OR}=2,022)$.

A Tabela 2 apresenta os fatores associados (sociodemográficos e familiares) às diferentes categorias de consumo em comparação aos adolescentes que não consumiram nenhuma substância.

\section{Uso de substância entre os adolescentes}

Entre os adolescentes que responderam às questões sobre consumo de drogas (919), 570 (62\%) não usaram nenhuma substância, 208 (22,6\%) usaram apenas álcool, 24 (2,6\%) apenas tabaco, $54(5,9 \%)$ álcool e tabaco e 63 (6,9\%) usaram alguma droga ilícita nos 30 dias anteriores à entrevista. As drogas ilícitas utilizadas foram: maconha $(\mathrm{n}=27 ; 2,9 \%)$, tranquilizantes $(\mathrm{n}=17 ; 1,8 \%)$, anfetaminas $(n=15 ; 1,6 \%)$, ecstasy $(n=10 ; 1,1 \%)$, inalantes $(n=10 ; 1,1 \%)$, cocaína $(n=8 ; 0,8 \%)$, alucinógenos ( $\mathrm{n}=4 ; 0,4 \%)$ e anabolizantes ( $\mathrm{n}=$ $4 ; 0,4 \%)$.
Os estudantes entre 15 e 18 anos apresentaram 2,7 vezes mais chances de fazer uso apenas de álcool ( $\mathrm{p}<0,001), 3$ vezes mais chances de usar álcool e tabaco ( $p<0,001)$ e 4,6 vezes mais chances de usar drogas ilícitas $(\mathrm{p}<0,001)$ do que os de 10 a 14 anos. Ter repetido o ano escolar também aumentou a chance dos adolescentes fazerem uso de álcool e tabaco $(\mathrm{p}=0,034 ; \mathrm{OR}=2,076)$ e drogas ilícitas $(\mathrm{p}<0,001 ; \mathrm{OR}=3,551)$.

Não houve associação entre a variável "pessoa com quem reside" e o consumo de álcool ( $\mathrm{p}=0,413)$, tabaco $(\mathrm{p}=0,449)$, álcool e tabaco ( $\mathrm{p}=0,843)$ e drogas ilícitas $(\mathrm{p}=0,384)$. O consumo de álcool ( $\mathrm{p}=0,657)$, álcool e tabaco ( $\mathrm{p}=$ $0,641)$ e drogas ilícitas $(\mathrm{p}=1,000)$ foi semelhante entre os adolescentes dos sexos feminino e masculino. O uso de tabaco foi mais frequente entre as meninas ( $\mathrm{p}=0,048$; $\mathrm{OR}=0,393)$. 
Frequência de consumo de substâncias segundo o relato de problemas familiares entre adolescentes de 50 escolas públicas estaduais de Jacareí e Diadema, São Paulo $(\mathrm{N}=965)$ *

\begin{tabular}{|c|c|c|c|c|c|c|c|c|}
\hline & \multicolumn{2}{|c|}{ Tabaco } & \multicolumn{2}{|c|}{ Álcool } & \multicolumn{2}{|c|}{$\begin{array}{c}\text { Álcool e } \\
\text { tabaco }\end{array}$} & \multicolumn{2}{|c|}{ Drogas ilícitas } \\
\hline & $\%$ & OR & $\%$ & OR & $\%$ & OR & $\%$ & OR \\
\hline \multicolumn{9}{|l|}{ Idade (anos) $(\mathrm{n}=828)$} \\
\hline $10-14$ & 25,0 & & 33,7 & & 35,7 & & 47,1 & \\
\hline $15-18$ & 75,0 & 1,7 & 66,3 & $2,7 * \star$ & 64,3 & $3,0 * \star$ & 52,9 & $4,6 * \star$ \\
\hline Repetiu o ano ( $n=912$ ) & 29,2 & 2,2 & 16,8 & 1,0 & 27,8 & $2,0 * \star \star$ & 39,7 & $3,5 * \star$ \\
\hline Relacionamento ruim $(n=904)$ & 20,8 & 1,8 & 16,2 & 1,4 & 30,0 & 4,1 ** & 29,3 & 2,7 ** \\
\hline Ter membro da família que usou maconha ou cocaína no último mês ( $n=912$ ) & 25,0 & $5,5 * \star \star$ & 8,7 & 1,1 & 15,3 & $2,9 \#$ & 22,8 & 4,4 ** \\
\hline Ter membro da família que usou álcool a ponto de causar problemas ( $\mathrm{n}=912$ ) & 25,0 & 1,3 & 28,0 & $2,1 * \star$ & 37,3 & $3,0 * \star$ & 43,9 & 3,6 ** \\
\hline Ter membro da família que foi preso no último mês $(n=910)$ & 20,8 & 2,5 & 12,1 & 1,4 & 10,2 & 1,3 & 31,6 & $4,8 * \star$ \\
\hline Tem discussões frequentes com os pais $(n=904)$ & 29,2 & 1,8 & 27,3 & $2,4 * \star$ & 35,6 & $3,3 * \star$ & 48,2 & $5,1 * \star$ \\
\hline Família dificilmente faz coisas juntas $(n=908)$ & 37,5 & 1,4 & 35,0 & $1,6 * \star \star$ & 39 & $2,3 * \star \star$ & 56,1 & $3,5 * \star$ \\
\hline Faltam regras claras, em casa, sobre o que pode ou não fazer $(n=909)$ & 6,6 & 1,8 & 34,3 & $1,5 \#$ & 11,5 & 1,4 & 13,3 & 1,5 \\
\hline Pais desconhecem o que gosta ou desgosta $(n=899)$ & 27,3 & 1,1 & 25,9 & 1,0 & 33,9 & 1,6 & 44,6 & $2,3 * \star \star$ \\
\hline Pais desconhecem o que pensa ou sente sobre coisas importantes para ele $(n=901)$ & 30,4 & 1,2 & 29,6 & 1,2 & 37,3 & $2,0 \#$ & 46,3 & 2,6 ** \\
\hline Pais brigam muito entre si $(n=911)$ & 20,8 & 1,5 & 18,8 & $1,8 * \star \star$ & 28,8 & 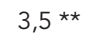 & 25,0 & $2,6 * \star \star$ \\
\hline Pais desconhecem onde está ou o que está fazendo ( $n=908)$ & 33,3 & 2,2 & 19,3 & 1,0 & 23,7 & 1,5 & 45,6 & $3,7 * \star$ \\
\hline Pais ficam fora de casa a maior parte do tempo $(n=915)$ & 37,5 & 1,3 & 36,1 & $1,5 * \star \star$ & 42,4 & $2,2 \star \star \star$ & 42,1 & $1,8 \#$ \\
\hline Sente que os pais não se importam/não cuidam dele $(n=914)$ & 16,7 & 1,8 & 13,5 & $2,0 * \star \star$ & 18,6 & 3,4 ** & 24,6 & $4,2 * \star$ \\
\hline Se sente infeliz onde vive $(n=910)$ & 25,0 & 1,7 & 16,8 & 1,0 & 25,4 & 2,0 & 21,1 & 1,3 \\
\hline Se sente em perigo em casa $(n=912)$ & 20,8 & $4,8 * \star \star$ & 6,7 & 0,9 & 15,3 & $3,7 \star \star \star$ & 10,5 & 1,6 \\
\hline Na maioria das festas que frequentam os pais estão ausentes $(n=909)$ & 41,7 & 1,4 & 41,0 & $1,6 * \star \star$ & 38,3 & 1,4 & $42,9 \#$ & 1,7 \\
\hline Passa a maior parte do tempo livre com os amigos $(n=910)$ & 56,5 & 1,6 & 49,5 & $1,4 \#$ & 55 & $2,1 \star \star \star$ & 59,6 & $2,0 * \star \star$ \\
\hline Realiza a maior parte das atividades de lazer sozinho $(n=899)$ & 29,2 & 1,9 & 16,7 & 0,8 & 32,2 & $2,4 * \star \star$ & 21,8 & 1,2 \\
\hline
\end{tabular}

OR: razão de chance.

* As OR referem-se à comparação com os adolescentes que não usaram as respectivas drogas em questão. Os valores não são ajustados;

** $\mathrm{p} \leq 0,001$;

$\star \star \star ~ p \leq 0,01$;

$\# p \leq 0,05$.

\section{Consumo de tabaco entre os adolescentes e problemas familiares}

Os adolescentes que usaram tabaco apresentaram 5,5 vezes mais chances de ter algum membro da família que usou maconha ou cocaína no mês anterior à entrevista $(p=0,001)$ e 4,8 vezes mais chances de se sentir em perigo em casa $(\mathrm{p}=$ 0,005 ) do que aqueles que não consumiram nenhuma substância.

\section{Consumo de álcool entre os adolescentes e problemas familiares}

Os adolescentes que consumiram álcool tiveram até 2 vezes mais chances de achar que os pais não se importam ou não cuidam deles ( $p=0,008)$, de não ter um bom relacionamento com os pais $(\mathrm{p}=0,007)$, dos pais brigarem muito entre si $(\mathrm{p}=$ $0,006)$, da família dificilmente fazer coisas juntos ( $p=0,005)$, de frequentar festas em que não há pais presentes $(p=0,004)$, de faltar regras claras sobre o que se pode ou não fazer $(p=0,034)$, dos pais ficarem fora a maior parte do tempo ( $\mathrm{p}=0,015)$ e de passar a maior parte do tempo livre com os amigos $(p=0,024)$ em comparação com os adolescentes que não usaram nenhuma substância. Também apresentaram 2,1 vezes mais chances de ter algum membro da família que usou álcool a ponto de causar problemas em casa, no trabalho ou com amigos ( $<<0,001)$ e 2,4 vezes mais chances de ter discussões frequentes com os pais $(\mathrm{p}<0,001)$. 
Consumo de álcool e tabaco entre os adolescentes e problemas familiares

Os adolescentes que fizeram uso de álcool e tabaco apresentaram entre 2 e 3 vezes mais chances da família dificilmente fazer coisas juntos $(\mathrm{p}=$ $0,005)$, dos pais desconhecerem o que eles pensam/sentem sobre coisas que os adolescentes consideram importantes ( $p=0,029)$, dos pais ficarem fora de casa a maior parte do tempo ( $\mathrm{p}=$ 0,007), de passar a maior parte do tempo livre com os amigos $(\mathrm{p}=0,010)$ e de realizar a maior parte das atividades de lazer sozinho $(p=0,007)$ em comparação com aqueles que não usaram nenhuma substância.

Também apresentaram entre 3 e 4 vezes mais chances de não avaliar a relação com os pais como boa ( $\mathrm{p}<0,001)$, de sentir-se em perigo em casa $(p=0,002)$, dos pais brigarem muito entre si ( $\mathrm{p}<0,001)$, de sentir que os pais não se importam/não cuidam deles ( $\mathrm{p}=0,001)$, de discutir frequentemente com os pais ( $p<0,001$ ), de ter pais que usaram álcool a ponto de ter problemas $(\mathrm{p}<$ $0,001)$ e de ter pais que fizeram uso de maconha ou cocaína $(p=0,017)$.

\section{Consumo de drogas ilícitas entre os} adolescentes e problemas familiares

Como pode ser visto na Tabela 2, os adolescentes que usaram alguma droga ilícita tiveram até 3 vezes mais chances de não avaliar a relação com os pais como boa ( $\mathrm{p}<0,001)$, dos pais desconhecerem o que eles gostam e desgostam $(p=0,002)$, dos pais desconhecerem o que eles pensam ou sentem sobre coisas que consideram importantes $(p=0,001)$, dos pais brigarem muito entre si ( $p=0,004)$, dos pais passarem a maior parte do tempo fora de casa $(\mathrm{p}=0,035)$ e de passar a maior parte do tempo livre com os amigos ( $p=0,009)$ em comparação com aqueles que não usaram nenhuma substância.

Também apresentaram entre 3 e 5 vezes mais chances de ter algum membro da família que fez uso de maconha ou cocaína ( $p<0,001)$, de ter pais que usaram álcool a ponto de ter problemas $(\mathrm{p}<0,001)$, de ter algum membro da família que foi preso no mês anterior à entrevista $(\mathrm{p}<$ $0,001)$, de discutir frequentemente com os pais ( $\mathrm{p}<0,001$ ), da família dificilmente fazer coisas juntos ( $p<0,000)$, dos pais não saberem onde eles estão ou o que estão fazendo $(\mathrm{p}<0,001) \mathrm{e}$ de sentir que os pais não se importam ou não cuidam deles $(\mathrm{p}<0,001)$.

A Tabela 3 mostra os resultados obtidos com a análise múltipla. A idade entre 15 e 18 anos foi o fator de maior associação com o uso de álcool entre os adolescentes $(\mathrm{p}<0,001)$, seguido por ter membro da família que usou álcool a ponto de ter prejuízos ( $\mathrm{p}=0,005)$, sentir que os pais não se importam ou não cuidam deles $(\mathrm{p}=0,014)$ e discutir frequentemente com os pais $(\mathrm{p}=0,042)$.

Em relação ao uso de tabaco, sentir que os pais não se importam ou não cuidam deles ( $\mathrm{p}=$ 0,017 ) foi o fator de maior associação, seguido por ter algum membro da família que usou maconha ou cocaína ( $p=0,018$ ). Para os adolescentes que usaram álcool e tabaco, os fatores de maior associação foram: não avaliar positivamente a relação que têm com os pais ( $p=0,001)$, ter entre 15 e 18 anos ( $p=0,003$ ), sentir que os pais não se importam ou não cuidam deles $(\mathrm{p}=0,006)$ e ter membro da família que usou álcool a ponto de ter prejuízos ( $\mathrm{p}=0,030)$.

Entre os adolescentes que usaram drogas ilícitas, a idade foi o fator de maior associação ( $\mathrm{p}<$ 0,001 ), seguido por discutir frequentemente com os pais $(p=0,005)$, passar a maior parte do tempo livre com os amigos ( $\mathrm{p}=0,005)$, sentir que os pais não se importam ou cuidam deles $(\mathrm{p}=0,007) \mathrm{e}$ ter membro da família que foi preso $(p=0,009)$.

\section{Categorias de consumo de substâncias e problemas familiares}

A Figura 1 mostra a média do número de problemas familiares relatados pelos adolescentes pertencentes às diferentes categorias de consumo. Os que usaram álcool ( $\mathrm{p}<0,001)$, tabaco $(\mathrm{p}=$ $0,001)$, álcool e tabaco $(\mathrm{p}<0,001)$ e drogas ilícitas $(\mathrm{p}<0,001)$ relataram ter mais problemas familiares do que aqueles que não usaram nenhuma substância. Os adolescentes que usaram álcool e tabaco $(\mathrm{p}=0,028)$ e drogas ilícitas $(\mathrm{p}<0,001)$ relataram ter mais problemas familiares do que aqueles que usaram apenas álcool. Não houve diferença entre os adolescentes que usaram álcool e tabaco e aqueles que usaram drogas ilícitas $(\mathrm{p}=0,449)$ em relação à média de problemas familiares.

\section{Discussão}

A classificação do consumo de drogas em cinco categorias (não uso, uso somente de álcool, uso somente de tabaco, uso de álcool e tabaco, e uso de drogas ilícitas) vem ao encontro de estudo recente que discute padrões de início e evolução do consumo e associações com diferentes probabilidades de problemas sociais, familiares, escolares, legais e de saúde associados a este consumo ${ }^{14}$.

Corroborando a literatura, adolescentes mais velhos tiveram mais chances de usar substâncias psicoativas do que os mais novos 2,15. Também 
Análise múltipla do uso de álcool, tabaco e drogas ilícitas segundo relato de problemas familiares entre adolescentes de 50 escolas públicas estaduais de Jacareí e Diadema, São Paulo, Brasil ( $N=919)$.

\begin{tabular}{lccc}
\hline Categorização do uso de substância & Valor de p & OR & IC95\% \\
\hline Usou apenas álcool & & & \\
$\quad$ Idade entre 15 e 18 anos & $<0,001$ & 2,393 & $1,581-3,623$ \\
$\quad$ Ter membro da família que usa álcool a ponto de ter prejuízos & 0,005 & 1,903 & $1,215-2,978$ \\
$\quad$ Ter discussões frequentes com os pais & 0,042 & 1,642 & $1,017-2,649$ \\
$\quad$ Sentir que os pais não se importam ou cuidam dele & 0,014 & 2,232 & $1,178-4,226$ \\
Usou apenas tabaco & & \\
$\quad$ Ter membro da família que usou maconha ou cocaína & 0,018 & 4,972 & $1,322-18,698$ \\
$\quad$ Sentir que os pais não se importam ou cuidam dele & 0,017 & 4,697 & $1,318-16,742$ \\
Usou álcool e tabaco & & \\
Idade entre 15 e 18 anos & 0,003 & 2,747 & $1,404-5,376$ \\
$\quad$ Relacionamento com os pais & 0,001 & 3,690 & $1,687-8,069$ \\
$\quad$ Ter membro da família que usa álcool a ponto de ter prejuízos & 0,030 & 2,194 & $1,081-4,452$ \\
Sentir que os pais não se importam ou cuidam dele & 0,006 & 3,373 & $1,409-8,076$ \\
Drogas ilícitas & & & \\
$\quad$ Idade entre 15 e 18 anos & 0,000 & 4,649 & $2,345-9,214$ \\
$\quad$ Relacionamento com os pais & 0,090 & 2,102 & $0,890-4,963$ \\
Ter membro da família que foi preso & 0,009 & 3,068 & $1,318-7,139$ \\
Ter discussões frequentes com os pais & 0,005 & 2,866 & $1,369-6,000$ \\
Sentir que os pais não se importam ou cuidam dele & 0,007 & 3,575 & $1,428-8,952$ \\
Passar a maior parte das horas livres com os amigos & 0,005 & 2,704 & $1,343-5,444$ \\
\hline
\end{tabular}

IC95\%: intervalo de 95\% de confiança; OR: razão de chance.

Figura 1

Relação entre a média de problemas familiares e o consumo de substâncias entre os adolescentes.

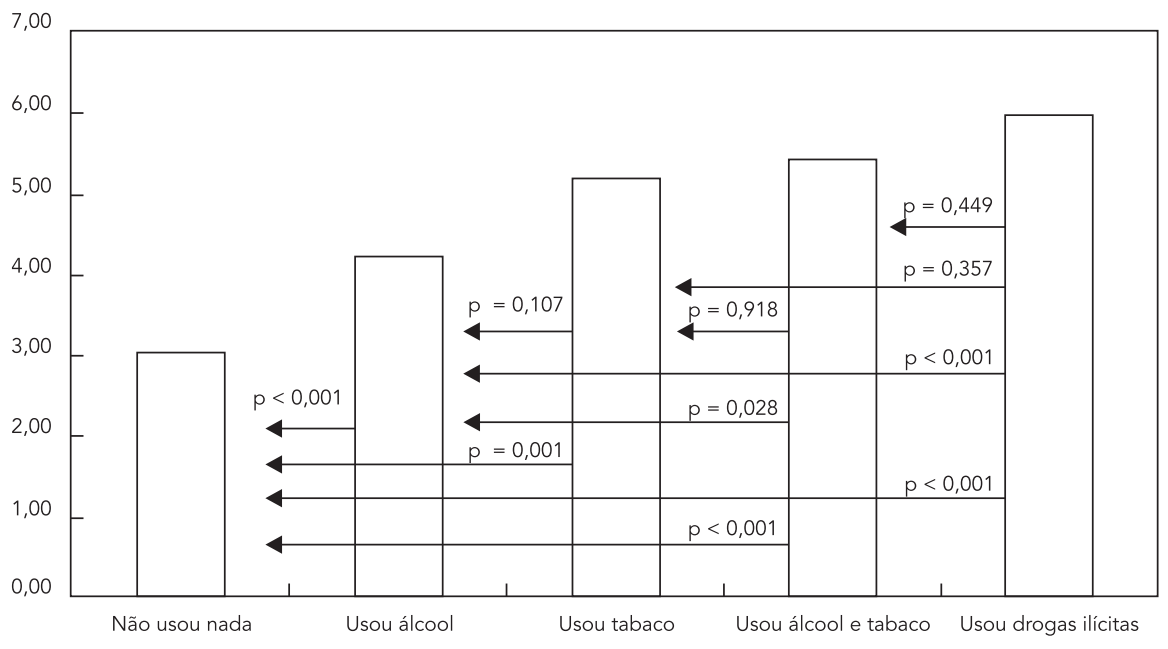


houve associação entre aumento da idade e maior chance de usar drogas ilícitas. Os mais velhos também apresentaram até duas vezes mais chances de usar mais de uma droga do que os mais jovens.

Os adolescentes que repetiram o ano escolar tiveram mais chances de usar álcool e outras drogas do que aqueles que não repetiram. Estudos mostram que repetência e evasão escolar são mais frequentes em estudantes que usam substâncias 16. Para os adolescentes que usaram álcool e tabaco e para os que usaram drogas ilícitas, os riscos de repetência foram duas e três e meia vezes maior, respectivamente, do que para aqueles que não usaram nenhuma substância.

O gênero é outra variável sociodemográfica frequentemente associada ao uso de álcool e drogas entre adolescentes. Em geral, o consumo tende a ser mais freqüente entre os meninos 2,7 , mas a diferença vem diminuindo nos últimos anos 1 . Neste trabalho não houve diferenças nas taxas de consumo entre meninos e meninas, exceto para o uso de tabaco que foi mais frequente entre as meninas, o que já havia ocorrido em outro estudo em nosso país 17. Uso de álcool, tabaco e drogas parece estar cada vez mais deixando de ser um comportamento essencialmente masculino em vários países, incluindo Brasil 17.

A associação, observada neste estudo, entre uso de álcool e drogas pelos pais e risco de uso destas substâncias pelos filhos tem sido explicada por fatores genéticos e ambientais 18. Além disso, pais que usam substâncias oferecem menos suporte, monitoramento e outros cuidados aos filhos. O uso de substâncias também poderia levar a discussões frequentes entre ambos, contribuindo para a falta de controle e dificultando a inserção de limites e disciplina 19.

$\mathrm{Na}$ análise multivariada, as variáveis "passar a maior parte das horas livres com amigos" e "ter membro da família que foi preso" mostraram-se associadas somente ao uso de drogas ilícitas, provavelmente apontando para um menor monitoramento dos pais em relação aos adolescentes. Também indicando tal distanciamento familiar, as razões de chances das associações entre uso da substância e a variável "sentir que os pais não se importam ou cuidam deles" são menores nas categorias uso de álcool e tabaco isoladamente, em relação às categorias uso de álcool e tabaco e drogas ilícitas, indicando um incremento da associação entre este problema familiar e o uso das duas drogas lícitas concomitantemente. Esse último padrão parece ser semelhante ao uso de drogas ilícitas. A falta de monitoramento tem sido apontada como um dos fatores familiares mais associados ao risco de uso de substâncias psicoativas por adolescentes 20 .
Também na análise multivariada, aspectos como repetência escolar e gênero não expressaram maior chance de uso de substâncias. De forma relevante, apenas o uso de tabaco não se associou à faixa etária mais velha, sugerindo o uso mais precoce desta substância nesta amostra.

Quando as médias de problemas familiares são analisadas (Figura 1), os adolescentes que usaram tabaco, álcool e tabaco, e drogas ilícitas não apresentaram diferenças significativas. Os usos isolados de álcool e de tabaco também tiveram médias de problemas no sistema familiar semelhantes. Portanto, aparentemente, o uso de tabaco ocupa uma posição intermediária entre as categorias somente álcool e as demais em termos de média de problemas.

Diversos fatores relacionados ao sistema familiar discutidos neste artigo têm sido apontados como indicadores de risco para o uso de substâncias entre os adolescentes. Todavia, até onde se pôde observar, não foi encontrado nenhum estudo que mostrasse que o uso de álcool e tabaco concomitantemente e de drogas ilícitas está associado a mais problemas familiares (mensurados por meio da média dos problemas referidos pelos adolescentes) do que o uso de álcool isoladamente. Esse é o principal achado deste trabalho. Ou seja, os adolescentes que fizeram uso de álcool e tabaco ( $\mathrm{p}=0,028)$ e drogas ilícitas $(\mathrm{p}<0,001)$ relataram ter mais problemas familiares do que aqueles que usaram apenas álcool.

O uso de álcool e tabaco esteve associado a prejuízos familiares significativos, semelhantes aos associados ao uso de drogas ilícitas. Ou seja, o consumo das duas drogas lícitas mais comuns parece ser mais grave do que o consumo de uma delas isoladamente. Essa associação merece atenção, já que há uma tendência em acreditar que o uso de álcool e tabaco é um comportamento esperado na adolescência. Essa percepção parece estar associada ao fato de serem drogas lícitas, à alta prevalência do uso em nossa sociedade e à expectativa que adolescentes contestem regras e limites 21 .

O fato do uso de álcool estar menos associado a problemas familiares que o uso de álcool e tabaco também pode indicar recentes mudanças sociais, caracterizadas por maior aceitação do uso e maior disponibilidade do álcool do que do tabaco. Revisão recente aponta para os bons resultados de campanhas educativas e sociais na redução do consumo de tabaco, mas, por outro lado, ressalta o baixo impacto e a inconsistência dos resultados de campanhas que abordam o consumo de álcool e drogas ilícitas 22 .

A alta frequencia de problemas familiares em estudantes que usaram alguma substância neste estudo indica que as estratégias de pre- 
venção devam conter intervenções nos adolescentes e nos familiares. Tal modelo tem mostrado bons resultados na prevenção e redução de problemas associados ao consumo de substâncias 23,24 .

\section{Limitações do estudo}

Este estudo, pela sua transversalidade, não foi capaz de avaliar se as associações encontradas são causais nem a possível direção da causalidade. O consumo de álcool e tabaco pode estar aumentando as chances dos adolescentes referirem problemas familiares ou os problemas familiares podem estar aumentando as chances de consumo destas substâncias. Problemas familiares e consumo de drogas podem, ainda, ser consequências de outros fatores de risco comuns aos dois problemas, como por exemplo, transtornos mentais. Neste trabalho, não realizamos uma avaliação dos transtornos mentais nos estudantes, e, portanto, deve-se considerar que as associações encontradas podem, pelo menos em parte, decorrer de transtornos psiquiátricos associados tanto aos problemas familiares quanto ao uso de substâncias. Vale ressaltar, entretanto, que o fato de todos os adolescentes estarem estudando em escolas regulares sugere que não haja transtornos mentais graves.

Vieses de amostragem (apenas 50 escolas em dois municípios do Estado de São Paulo foram avaliadas) e de autosseleção (adolescentes tinham o controle sobre a possibilidade de participação) não podem ser descartados. Esses fatos podem comprometer a generalização desses resultados para toda a população brasileira. Por outro lado, o tamanho da amostra (quase 1.000 adolescentes) é uma das virtudes deste trabalho. O presente estudo pode servir de estímulo para levantamentos nacionais sobre tema tão relevante.

\section{Resumo}

Esta pesquisa visou a avaliar a associação entre o consumo de substâncias (álcool, tabaco e drogas ilícitas) e problemas familiares numa amostra de 965 adolescentes em 50 escolas públicas de dois municípios do Estado de São Paulo, Brasil, em 2007. Foi utilizado o Drug Use Screening Inventory (DUSI) para a coleta de dados. O uso de álcool, tabaco e drogas ilícitas foi associado à avaliação negativa da relação familiar, à falta de suporte/monitoramento e ao uso de substâncias por familiares $(p<0,05)$. Os estudantes que relataram ter feito uso de substâncias apresentaram mais problemas familiares do que aqueles que não consumiram nenhuma substância $(p<0,001)$. Os adolescentes que usaram álcool e tabaco $(p=0,028)$ e drogas ilícitas $(p<0,001)$ relataram ter mais problemas familiares do que aqueles que usaram apenas álcool. Os resultados apontam para a importância de se ficar atento ao consumo de álcool e tabaco entre os adolescentes, já que o relato do consumo das duas substâncias esteve associado a prejuízos familiares significativos, semelhantes ao uso de drogas ilícitas.

Bebidas Alcoólicas; Tabaco; Drogas Ilícitas; Relações Familiares; Adolescente

\section{Colaboradores}

A. Malbergier participou na concepção e planejamento da pesquisa, coordenação dos pesquisadores, análise e interpretação dos dados e elaboração do artigo. L. R. D. Cardoso participou na concepção e planejamento da pesquisa, análise e coleta dos dados, coordenação do trabalho de campo e elaboração do artigo. R. A. Amaral participou na coleta, análise e interpretação dos dados, e elaboração do artigo.

\section{Agradecimentos}

Organização das Nações Unidas para a Educação, Ciência e Cultura (UNESCO); Secretaria da Educação do Estado de Estado de São Paulo; Secretaria Nacional de Políticas Sobre Drogas. 


\section{Referências}

1. Brook DW, Brook JS, Rubenstone E, Zhang C, Gerochi C. Cigarette smoking in the adolescent children of drug-abusing fathers. Pediatrics 2006; 117:1339-47.

2. Galduróz JC, Sanchez ZVDM, Opaleye ES, Noto AR, Fonseca AM, Gomes PLS, et al. Fatores associados ao uso pesado de álcool entre estudantes das capitais brasileiras. Rev Saúde Pública 2010; 44:267-73.

3. Morihisa RS, Barroso LP, Scivoletto S. Labeling disorder - the relationship between conduct problems and drug use in adolescents. Rev Bras Psiquiatr 2007; 29:308-14.

4. Catanzaro SJ, Laurent J. Perceived family support, negative mood regulation expectancies, coping, and adolescent alcohol use: evidence of mediation and moderation effects. Addict Behav 2004; 29:1779-97.

5. Kliewer W, Murrelle L. Risk and protective factors for adolescent substance use: findings from a study in selected Central American countries. J Adolescent Health 2007; 40:448-55.

6. Kendler KS, Schmitt E, Aggen SH, Prescott CA. Genetic and environmental influences on alcohol, caffeine, cannabis, and nicotine use from early adolescence to middle adulthood. Arch Gen Psychiatry 2008; 65:674-82.

7. Poulin C, Hand D, Boudreau B, Santor D. Gender differences in the association between substance use and elevated depressive symptoms in a general adolescent population. Addiction 2005; 100:525-35.

8. Sanchez ZVDM, Oliveira LG, Nappo SA. Razões para o não-uso de drogas ilícitas entre jovens em situação de risco. Rev Saúde Pública 2005; 39: 599-605.

9. Dall'Agnol MM, Fassa AC, Facchini LA. Child and adolescent labor and smoking: a cross-sectional study in southern Brazil. Cad Saúde Pública 2011; 27:46-56.

10. Souza DPO, Areco KN, Silveira Filho DX. Álcool e alcoolismo entre adolescentes da rede estadual de ensino de Cuiabá, Mato Grosso. Rev Saúde Pública 2005; 39:585-92.

11. Vieira PC, Aerts DR, Freddo SL, Bittencourt A, Monteiro L. Alcohol, tobacco, and other drug use by teenage students in a city in Southern Brazil. Cad Saúde Pública 2008; 24:2487-98.

12. De Micheli D, Formigoni MLOS. Screening of drug use in a teenage Brazilian sample using the Drug Use Screening Inventory (DUSI). Addict Behav 2000; 25:683-91.

13. De Micheli D, Formigoni MLOS. Psychometrics properties of the Brazilian version of DUSI (Drug Use Screening Inventory). Alcohol Clin Exp Res 2002; 26:1523-8.
14. Degenhardt L, Chiu WT, Conway K, Dierker L, Glantz M, Kalaydjian A, et al. Does the 'gateway' matter? Associations between the order of drug use initiation and the development of drug dependence in the National Comorbidity Study Replication. Psychol Med 2009; 39:157-67.

15. Centro Brasileiro de Informações Sobre Drogas Psicotrópicas. V levantamento nacional sobre o consumo de drogas psicotrópicas entre estudantes do ensino fundamental e médio da rede pública de ensino nas 27 capitais brasileiras. http:// www.unifesp.br/dpsicobio/cebrid/ (acessado em 20/Set/2008).

16. Bahls FRC, Ingbermann YK. Desenvolvimento escolar e abuso de drogas na adolescência. Estud Psicol (Campinas) 2005; 22:395-402.

17. Horta RL, Horta BL, Pinheiro RT, Morales B, Strey MN. Tobacco, alcohol, and drug use by teenagers in Pelotas, Rio Grande do Sul State, Brazil: a gender approach. Cad Saúde Pública 2007; 23:775-83.

18. Xian H, Scherrer JF, Grant JD, Eisen SA, True WR, Jacob T, et al. Genetic and environmental contributions to nicotine, alcohol and cannabis dependence in male twins. Addiction 2008; 103:1391-8.

19. Mathews IS, Pillon SC. Factores protectores y de riesgo asociados al uso de alcohol en adolescentes hijos de padre alcohólico, en el Perú. Rev Latinoam Enferm 2004; 12:359-68.

20. Tobler AL, Komro KA. Trajectories or parental monitoring and communication and effects on drug use among urban young adolescents. J Adolescent Health 2010; 46:560-8.

21. Marques CPR, Cruz MS. O adolescente e o uso de drogas. Rev Bras Psiquiatr 2000; 22 Suppl II:32-6.

22. Wakefield MA, Loken B, Hornik RC. Use of mass media campaigns to change health behaviour. Lancet 2010; 376:1261-71.

23. Griffin KW, Botwin GJ Evidence-based interventions for preventing substance use disorders in adolescents. Child Adolesc Psychiatr Clin N Am 2010; 19:505-26

24. Wagner KD, Ritt-Olson A, Chou CP, Pokhrel P, Duan L, Baezconde-Garbanati L, et al. Associations between family structure, family functioning, and substance use among Hispanic/Latino adolescents. Psychol Addict Behav 2010; 24:98-108.

Recebido em 21/Abr/2011

Versão final reapresentada em 27/Out/2011

Aprovado em 05/Jan/2012 